\title{
A NOTE ON REPRESENTATION BY POLYGONAL NUMBERS
}

\author{
L. W. GRIFFITHS
}

1. Introduction. The universal functions of polygonal numbers of order $m+2$ were determined in an earlier paper. ${ }^{1}$ In each universal function the number $n$ of variables was at most $m+2$. The minimum value $N$ of $n$, for each integer $m \geqq 3$, will be proved in this paper to be the integer $N$ defined by (3). It will also be proved that there is a unique universal function having $n=N$ if and only if $m=3,4$, $2^{N-2}-2,2^{N-2}-1$. A universal function having $n=N$ is given by the integers (4). At least one universal function different from this function is given by the integers (6) if $m \neq 3,4,2^{N-2}-2,2^{N-2}-1$, and if (7) and (8) hold.

2. Proofs. In the notations of the paper to which reference has been made $m$ was an arbitrary but fixed integer greater than or equal to 3 . The coefficients $a_{1}, a_{2}, \cdots, a_{n}$ in the universal functions were positive integers to be determined, and $1 \leqq a_{1} \leqq \cdots \leqq a_{n}$. Also, by definition, $w_{k}=a_{1}+\cdots+a_{k}(1 \leqq k \leqq n)$. It was proved that no function is universal if $w_{n}<m+2$, and that if $w_{n}=m+2$ then the function $f$ is universal if and only if $f$ is one of the following:

$$
\begin{array}{r}
(1,1,1,1,1) \text { or }(1,1,1,2), \quad \text { with } m=3 \text { and } w_{n}=m+2, \\
\left(1,1,1, a_{4}, \cdots, a_{n}\right), w_{n}=m+2>5, a_{k} \leqq w_{k-1}-1(4 \leqq k \leqq n), \\
\text { but } n>5 \text { and } a_{5} \neq 3 \text { if } a_{4}=1 .
\end{array}
$$

Thus $N=4$ if $m=3$. It will be proved that if $m>3$ then the minimum $m$ is the integer $N$ uniquely defined by

$$
2^{N-3}-1<m \leqq 2^{N-2}-1 .
$$

Consider the sequence of integers $1,1,1,2,2^{2}, \cdots, 2^{i-3}, \cdots$ in which the $i$ th term is $2^{i-3}$ if $i \geqq 3$. The sum of the first $i$ terms is $2^{i-2}+1$ if $i \geqq 3$. Now let $f$ be a universal function which satisfies (2). Then it is easily proved by induction that $a_{k} \leqq 2^{k-3}$ and $w_{k} \leqq 2^{k-2}+1$ $(3 \leqq k \leqq n)$. Hence if $n<N$ this would imply in particular that $m+2=w_{n} \leqq 2^{n-2}+1 \leqq 2^{N-3}+1$. This contradiction of (3) when $n<N$ shows that $n \geqq N$. Furthermore $N \geqq 5$ since $m>3$ in (3).

Presented to the Society, April 11, 1941, under the title The minimum number of variables in universal functions of polygonal numbers; received by the editors April 11, 1941.

${ }^{1} \mathrm{~L}$. W. Griffiths, $A$ generalization of the Fermat theorem on polygonal numbers, Annals of Mathematics, (2), vol. 31 (1930), pp. 1-12. 
That $N$ is the minimum value of $n$ will be proved now, by exhibiting a function satisfying (2) and having $n=N$. This function has as its coefficients the integers

$$
1,1,1,2,2^{2}, \cdots, 2^{N-4}, m+1-2^{N-3}
$$

after they have been arranged in order of increasing magnitude. This can be done because by (3) with $N \geqq 5$ there is a unique integer $J$ such that $0 \leqq J \leqq N-3$ and $2^{J} \leqq m+1-2^{N-3}<2^{J+1}$. It is easily verified that the rearranged integers (4) satisfy all the conditions in (2).

The function defined by (4) is the only function satisfying (2) and having $n=N$ if $m=4$. This is also true if

$$
m=2^{N-2}-1 \text { or } 2^{N-2}-2 .
$$

Then $m+1-2^{N-3}$ is $2^{N-3}$ or $2^{N-3}-1$ respectively, and the integers (4) require no rearrangement. Now if $f$ is a function which satisfies (2) with $n=N$ and is different from this exhibited function, then there is a coefficient $a_{j}<2^{j-3}$ with $j \leqq N-1$. Then $w_{N-1} \leqq 2^{N-3}$ and $a_{N} \leqq 2^{N-3}$ -1 , and hence $m+2=w_{N} \leqq 2^{N-2}-1$. This contradicts (5).

The function defined by (4) is not the only function satisfying (2) and having $n=N$ if $m \neq 4,2^{N-2}-2,2^{N-2}-1$. This will be proved by finding conditions on the integers $b$ and $c$ which are necessary and sufficient that the sequence

$$
1,1,1,2,2^{2}, \cdots, 2^{N-5}, b, c
$$

yields, after rearrangement in order of increasing magnitude, the coefficients of a function satisfying (2). Then values of $b$ and $c$ will be exhibited such that these conditions are satisfied and that the set of integers (6) is not the set (4).

Let $b$ be any integer such that

$$
\left(m+1-2^{N-3}\right) / 2 \leqq b \leqq\left(m+1-2^{N-4}\right) / 2, b \leqq 2^{N-4},
$$

and let $c$ be defined by

$$
c=m+1-2^{N-4}-b .
$$

It is easily seen that (8) is equivalent to the sum of the integers (6) being $m+2$, and that the second inequality in (7) is equivalent to $b \leqq c$. If in $b \leqq c$ the integer $b$ is replaced by its value from (8), and if (3) is applied, it is seen that $c \geqq 1+2^{N-5}$. Hence $c$ is the last integer after rearrangement of (6). Now the first inequality in (7) is equivalent, by (8), to the fact that $c+1$ is at most the sum of the preceding integers in (6). By the last inequality in (7) it is easily verified that the rearranged integers (6) satisfy the remaining conditions in (2). 
Now, if $m$ is odd, then $\left(m+1-2^{N-3}\right) / 2$ is an integer which may be taken as the value of $b$ since it satisfies the conditions (7). It is easily seen that the set (6), in which $b=\left(m+1-2^{N-3}\right) / 2$ and $c$ is determined by (8), is not the set (4). However, if $m$ is even and not equal to $2^{N-3}$, then $b=\left(m+2-2^{N-3}\right) / 2$ and $c$ determined by (8) are integers which satisfy (7) and yield a set (6) which is not the set (4). But if $m=2^{N-3}$, then $N \geqq 6$ and $b=2$ satisfies the conditions (7) on $b$ and yields a set (6) which is not the set (4).

An interesting choice of integers $b$ and $c$ is that given by $b=\left(m+1-2^{N-4}\right) / 2$ if $m$ is odd and less than or equal to $\left(2^{N-4}+2^{N-3}-1\right)$, but by $b=\left(m-2^{N-4}\right) / 2$ if $m$ is even and less than or equal to $\left(2^{N-4}+2^{N-3}\right)$. Then (6) require no rearrangement, and $c$ is respectively $b$ or $b+1$. The resulting integers (6) differ from (4) when $m \neq 2^{N-4}+2^{N-3}-1,2^{N-4}+2^{N-3}$.

NORTHWESTERN UNIVERSITY

\section{HAUSDORFF METHODS OF SUMMATION WHICH INCLUDE ALL OF THE CESȦRO METHODS}

\section{H. L. GARABEDIAN}

1. Introduction. The transformation ${ }^{1}$

$$
\sigma_{m}=\sum_{n=0}^{m} C_{m, n} \Delta^{m-n} c_{n} \cdot s_{n},
$$

where $c_{n}=\int_{0}^{1} u^{n} d \phi(u)$ and $\left\{s_{n}\right\}$ is a given sequence, defines a regular method of summation of the sequence $\left\{s_{n}\right\}$ provided that $\phi(u)$ is of bounded variation on the interval $0 \leqq u \leqq 1$, continuous at $u=0$, and

$$
\phi(u)=\left\{\begin{array}{l}
0 \quad \text { if } u=0, \\
1 \text { if } u=1, \\
\frac{1}{2}[\phi(u-0)+\phi(u+0)] \text { if } 0 \leqq u<1 .
\end{array}\right.
$$

If these conditions of regularity are fulfilled the sequence $\left\{c_{n}\right\}$ is said to be a regular moment sequence (briefly a regular sequence), the mass function $\phi(u)$ is said to be a regular mass function, and the method of summation involved is called a Hausdorff method of summation ([1] or [2]) and is designated by the symbol $[H, \phi(u)]$.

Presented to the Society, February 22, 1941; received by the editors April 8, 1941.

1 To define the symbolism used here we write $C_{m, n}=m(m-1) \cdots(m-n+1) / n !$, $C_{m, 0}=1 ; \Delta^{i} x_{j}=x_{j}-C_{i, 1} x_{j+1}+C_{i, 2} x_{j+2}+\cdots$. 